\title{
LUNDBERG-TYPE BOUNDS FOR THE JOINT DISTRIBUTION \\ OF SURPLUS IMMEDIATELY BEFORE AND AT RUIN UNDER A MARKOV-MODULATED RISK MODEL
}

\author{
BY
}

\author{
AndRew C.Y. NG ANd Hailiang YANG
}

\begin{abstract}
In this paper, we consider a Markov-modulated risk model (also called Markovian regime switching insurance risk model). Follow Asmussen (2000, 2003), by using the theory of Markov additive process, an exponential martingale is constructed and Lundberg-type upper bounds for the joint distribution of surplus immediately before and at ruin are obtained. As a natural corollary, bounds for the distribution of the deficit at ruin are obtained. We also present some numerical results to illustrate the tightness of the bound obtained in this paper.
\end{abstract}

\section{KEYWORDS}

Markov-modulated risk model, joint distribution of surplus immediately before and at ruin, change of probability measure, exponential martingale, Lundbergtype bounds.

\section{INTRODUCTION}

In the classical insurance risk model, compound Poisson process is used to model the surplus process. There is a huge amount of literature devoted to the generalization of the classical model in different ways. For more detailed discussions, see Gerber (1979), Grandell (1990), Rolski et al. (1999), Asmussen (2000) and the references therein.

Asmussen (1989) proposed a Markov-modulated risk model; and ruin probability was studied under the proposed model. This model is also called Markovian regime switching model in the finance and actuarial science literature. This model can capture the feature that insurance policies may need to change if economical or political environment changes.

The deficit at ruin has obvious financial impact to the insurance company if ruin has occurred. The distribution of surplus immediately before ruin provides us some idea about the company's financial situation before ruin is going to happen, therefore it is also an interesting quantity to study. The surplus at ruin, the surplus immediately before ruin and the joint distribution of the surplus 
immediately before and at ruin have been investigated by many authors recently. Gerber, Goovaerts and Kaas (1987) considered the distribution of the severity of ruin for the classical compound Poisson risk model. In their paper, an integral equation satisfied by the distribution of the severity of ruin was obtained. In the case of claim sizes following a mixture of exponential distributions or a mixture of Gamma distributions, closed form solutions were obtained. Later, Dufresne and Gerber (1988) introduced the distribution of the surplus immediately before ruin in the compound Poisson model. Similar results to those in Gerber, Goovaerts and Kaas (1987) were obtained in that paper. Dickson (1992) used a different way to deal with the distribution of surplus immediately before ruin. Dickson and Dos Reis (1994) extended the method of Dickson (1992) by using dual events to explain the relationship between the density of the surplus immediately before ruin, and the joint density of the surplus immediately before ruin and the severity of ruin. Schmidli (1999) considered the compound Poisson model with positive, negative or zero loading. Explicit expressions for the distributions of the surplus prior and at ruin were given in terms of ruin probability. Some asymptotic results for these distributions were obtained. Gerber and Shiu $(1997,1998)$ examined the joint distribution of the time of ruin, the surplus immediately before ruin and the deficit at ruin. They showed that the expected discounted penalty, considered as a function of the initial surplus, satisfies a renewal equation. Explicit solutions were obtained for some special cases. Gerber and Shiu (2005) studied the expected discounted penalty at ruin in a Sparre Andersen model and an integro-differential equation was obtained; the solution of this equation was given in terms of Laplace transforms. A closed form expression for the joint distribution of surplus immediately before and at ruin was obtained when the initial surplus is zero. In $\mathrm{Ng}$ and Yang (2005), the closed form solutions for the joint distribution of surplus immediately before and at ruin when the initial surplus is zero or when the claim size distributions are phase-type distributed are obtained under the Markovmodulated risk model.

In this paper, using the approach in Asmussen (2000, 2003), Lundberg-type upper bounds for the joint distribution are obtained. As some natural corollaries, bounds for the distribution of the deficit at ruin are obtained. We also present some numerical results to illustrate the tightness of the bound obtained in this paper.

\section{THE INSURANCE RISK MODEL}

Let $\left\{J_{t}\right\}_{t \geq 0}$ be a homogenous continuous-time Markov chain taking values in a finite set $\mathcal{M}=\{1,2, \ldots, d\}$ with generator $\boldsymbol{\Lambda}=\left[\lambda_{i j}\right]$. $\boldsymbol{\Lambda}$ is assumed to be irreducible with stationary distribution $\pi=\left(\pi_{1}, \pi_{2}, \ldots, \pi_{d}\right)$. In this paper, only exponentially bounded claim distributions, i.e. small claims, are considered. When $J_{t}=i$, the claim size distribution is $B_{i}$ with moment generating function $\hat{B}_{i}(s)$ and mean $\mu_{i}$, the arrival intensity is $\beta_{i}$ and the premium rate is $c_{i}$. Claims at different regimes are independent, and claims at the same regime are i.i.d. The initial surplus is $u \geq 0$.

A way to describe $R_{t}$ precisely is to first set up $d$ independent risk process $\left\{R_{t}^{l}\right\},\left\{R_{t}^{2}\right\}, \ldots,\left\{R_{t}^{d}\right\}$. Each $\left\{R_{t}^{i}\right\}$ is a classical compound Poisson risk process with 
premium rate $c_{i}$, claim arrival rate $\beta_{i}$, claim sizes distribution $B_{i}$ and zero initial surplus. Then the risk process $\left\{R_{t}\right\}$ is given by

$$
R_{t}=u+\sum_{i=1}^{d} \int_{0}^{t} 1\left(J_{s}=i\right) \mathrm{d} R_{s}^{i}
$$

where $1(A)$ is the indicator function of event $A$. We then define the claim surplus process $\left\{S_{t}\right\}$ as

$$
S_{t}=u-R_{t}
$$

This is the same model as in Asmussen (1989).

Following the proof of Theorem 12.3.2 of Rolski et al. (1999), it is easy to show that

$$
\lim _{t \rightarrow \infty} \frac{R_{t}}{t}=\sum_{i=1}^{d} \pi_{i}\left(c_{i}-\beta_{i} \mu_{i}\right) .
$$

Let $\mathbb{P}_{i}(\cdot)=\mathbb{P}\left(\cdot \mid J_{0}=i\right)$. From the above, the net profit condition, that is, the condition such that the ruin probability is strictly less than one starting with any state of economy, is

$$
\sum_{i=1}^{d} \pi_{i}\left(c_{i}-\beta_{i} \mu_{i}\right)>0
$$

Let $\tau(u)=\inf \left\{t: S_{t}>u\right\}=\inf \left\{t: R_{t}<0\right\}$ be the time of ruin with initial surplus $u$. For $i, j \in \mathcal{M}, u, x, y \geq 0$, let

$$
F_{i}(u, x, y)=\mathbb{P}_{i}\left(\tau(u)<\infty, R_{\tau(u)^{-}} \leq x, R_{\tau(u)} \geq-y \mid R_{0}=u\right)
$$

be the joint distribution of surplus immediately before and at ruin with initial surplus $u$ and initial state of economy $i$. The joint distribution of surplus immediately before and at ruin with initial surplus $u$ and initial state of economy $i$ and fixing the state of economy at the time of ruin to be $j$ is denoted by

$$
F_{i j}(u, x, y)=\mathbb{P}_{i}\left(\tau(u)<\infty, J_{\tau(u)}=j, R_{\tau(u)^{-}} \leq x, R_{\tau(u)} \geq-y \mid R_{0}=u\right) .
$$

We assume in the following that the net profit condition (2) holds and $c_{i}=1$ for all $i \in \mathcal{M}$ since we only deal with events in infinite horizon. Indeed for any sets of premium rate $\left\{c_{i}\right\}_{i \in \mathfrak{M}}$, the transformation

$$
\tilde{\lambda}_{i j}=\frac{\lambda_{i j}}{c_{i}}, \quad \tilde{\beta}_{i}=\frac{\beta_{i}}{c_{i}}, \quad \tilde{c}_{i}=1
$$

yields a process $\left\{\tilde{S}_{t}\right\}$ such that the joint distributions of the surplus before and after ruin with initial surplus $u$ for the corresponding $\left\{\tilde{R}_{t}\right\}$ and $\left\{R_{t}\right\}$ are the same. 


\section{Change of PRobability MEASURE}

Gerber $(1973,1979)$ advocated the use of martingales in ruin theory. By using martingale methods, Lundberg bounds for ruin probabilities in many insurance risk models, including the classical model, the Sparre Andersen model, the Markov-modulated risk model and the periodic risk model, can be obtained.

Let $E$ be a complete and separable space equipped with the natural filtration $\left\{\mathcal{F}_{t}\right\}_{t \geq 0}$ on the space $D_{E}$ of all real-valued functions which are right-continuous with left limit and Borel sigma-field $\mathcal{F}$, and let $\mathbb{P}$ be a given probability measure on $\left(D_{E}, \mathcal{F}\right)$. Suppose that $\left\{M_{t}\right\}_{t \geq 0}$ is a non-negative martingale with respect to $\left(\left\{\mathcal{F}_{t}\right\}_{t \geq 0}, \mathbb{P}\right)$ and $\mathbb{E} M_{0}=1$. Denote $\mathbb{E}[X 1(A)]$ by $\mathbb{E}[X ; A]$.

Since the bivariate process $\left\{\left(J_{t}, S_{t}\right)\right\}_{t \geq 0}$ is Markov, one can construct a martingale by the theory of Markov process. Asmussen (2000) constructed an exponential martingale for the Markov-modulated risk process by using the theory of Markov additive process. For general theory of Markov additive process, one can refer to Asmussen (2003). Consider the probability space $\left(\Omega,\left\{\mathcal{F}_{t}\right\}, \mathbb{P}_{i}\right)$, where $\mathcal{F}_{t}=\sigma\left\{\left(J_{v}, S_{v}\right): v \leq t\right\}$ is the $\sigma$-field generated by the Markov-modulated risk process. Let $s_{0}$ be chosen such that the moment generating functions $\hat{B}_{i}\left(s_{0}\right)<\infty$ for all $i \in \mathcal{M}$. Let $\boldsymbol{K}(\alpha)=\boldsymbol{\Lambda}+\operatorname{diag}\left(\beta_{i}\left(\hat{B}_{i}(\alpha)-1\right)\right)-\alpha \boldsymbol{I}$. By the PerronFrobenius thoerem (see, for example, Corollary A4.8 of Asmussen (2000)), the eigenvalue $\kappa(\alpha)$ of $\boldsymbol{K}(\alpha)$ with maximal real part is simple and real, and the corresponding right eigenvector $\boldsymbol{h}^{(\alpha)}$ can be chosen with all entries strictly positive. Here the normalization is $\boldsymbol{\pi} \boldsymbol{h}^{(\alpha)}=1$.

It can be shown that $\left\{M_{t}\right\}_{t \geq 0}$ defined by

$$
M_{t}=\frac{h_{J_{t}}^{(\alpha)}}{h_{J_{0}}^{(\alpha)}} e^{\alpha S_{t}-t \kappa(\alpha)}
$$

is a non-negative martingale with $\mathbb{E}_{i} M_{0}=1$ and $\mathbb{P}_{i}^{(\alpha)}$ defined by $\mathbb{P}_{i}^{(\alpha)}(A)=\mathbb{E}_{i}\left[M_{i}\right.$; $A]$ for any $A \in \mathcal{F}_{t}$ is a probability measure.

Under $\mathbb{P}_{i}^{(\alpha)},\left\{R_{t}\right\}_{t \geq 0}$ is still a Markov-modulated risk process with intensity matrix $\Lambda^{(\alpha)}=\left[\lambda_{i j}^{(\alpha)}\right]$, claim arrival rates $\beta_{j}^{(\alpha)}$ and claim size distributions $B_{j}^{(\alpha)}(x)$ for all $j \in \mathcal{M}$ where

$$
\begin{gathered}
\lambda_{i j}^{(\alpha)}= \begin{cases}\lambda_{i j} \frac{h_{j}^{(\alpha)}}{h_{i}^{(\alpha)}} & i \neq j \\
\lambda_{i i}+\beta_{i}\left(\hat{B}_{i}(\alpha)-1\right)-\kappa(\alpha)-\alpha & i=j,\end{cases} \\
\beta_{j}^{(\alpha)}=\beta_{j} \hat{B}_{j}(\alpha), \text { and } \\
B_{j}^{(\alpha)}(x)=\frac{1}{\hat{B}_{j}(\alpha)} \int_{0}^{x} e^{\alpha z} B_{j}(\mathrm{~d} z) .
\end{gathered}
$$

Also, $\kappa^{(\alpha)}(\theta)=\kappa(\alpha+\theta)-\kappa(\alpha)$. 
From Theorem 12.3.4 of Rolski et al. (1999), $\kappa(\alpha)$ is convex on $\left(-\infty, s_{0}\right)$ and

$$
\kappa^{\prime}(0)=-\left(1-\sum_{j \in \mathscr{M}} \pi_{j} \beta_{j} \mu_{j}\right)<0
$$

by the net profit condition (2), hence there may exist a unique positive solution $\gamma$ of the equation $\kappa(\alpha)=0$ and $\gamma$ is the adjustment coefficient.

To obtain an exponential bound for the joint distribution of surplus immediately before and at ruin, we use the probability measure $\mathbb{P}_{L, i}=\mathbb{P}_{i}^{(\gamma)}$. By differentiating with respect to $s, \kappa^{(\theta)^{\prime}}(s)=\kappa^{\prime}(s+\theta)$ and hence $\kappa^{(\gamma)^{\prime}}(0)=\kappa^{\prime}(\gamma)>0$ by the convexity of $\kappa(s)$. Then by

$$
\kappa^{(\gamma)^{\prime}}(0)=-\left(1-\sum_{j \in \mathcal{M}} \pi_{j}^{(\gamma)} \beta_{j}^{(\gamma)} \mu_{j}^{(\gamma)}\right),
$$

it is obvious that

$$
1-\sum_{j \in E} \pi_{j}^{(\gamma)} \beta_{j}^{(\gamma)} \mu_{j}^{(\gamma)}<0
$$

Hence the net profit condition does not hold under $\mathbb{P}_{L, i}$ and $\mathbb{P}_{L, i}(\tau(u)<\infty)=1$ for any $u \geq 0, i \in \mathcal{M}$.

In the following the adjustment coefficient $\gamma$ is assumed to exist. Let $\boldsymbol{h}=$ $\boldsymbol{h}^{(\gamma)}$ and $B_{L, j}(x)=B_{j}^{(\gamma)}(x)$.

\section{LUNDBERG BOUNDS}

In this section we shall derive exponential upper bounds for the joint distribution of surplus immediately before and at ruin and the distribution of the deficit at ruin using change of probability measure.

Theorem 1. Assume the net profit condition (2) holds and the adjustment coefficient $\gamma$ exists. The Lundberg-type upper bound for the joint distribution of surplus immediately before and at ruin starting with initial surplus $u$, state of economy $i$ and ruin in state of economy $j$ is given by

$$
F_{i j}(u, x, y) \leq \frac{h_{i}}{h_{j}} e^{-\gamma u} \sup _{0 \leq v \leq x} \frac{B_{j}(v+y)-B_{j}(v)}{\int_{v}^{\infty} e^{\gamma(z-v)} B_{j}(\mathrm{~d} z)}
$$

and the Lundberg-type upper bound for the joint distribution of surplus immediately before and at ruin starting with initial surplus $u$ and state of economy $i$ is given by

$$
F_{i}(u, x, y) \leq h_{i} e^{-\gamma u} \max _{j \in \mathcal{M}} \frac{1}{h_{j}} \sup _{0 \leq v \leq x} \frac{B_{j}(v+y)-B_{j}(v)}{\int_{v}^{\infty} e^{\gamma(z-v)} B_{j}(\mathrm{~d} z)}
$$


Proof: Let $\xi(u)=S_{\tau(u)}-u=\left|R_{\tau(u)}\right|$ be the overshoot above level $u$ when ruin occurs,

$$
\begin{aligned}
& F_{i j}(u, x, y) \\
= & \mathbb{P}_{i}\left(\tau(u)<\infty, J_{\tau(u)}=j, R_{\tau(u)-} \leq x, R_{\tau(u)} \geq-y\right) \\
= & h_{i} e^{-\gamma u} \mathbb{E}_{L, i}\left[\frac{e^{-\gamma \xi}(u)}{h_{J_{\tau(u)}}} ; J_{\tau(u)}=j, R_{\tau(u)-} \leq x, R_{\tau(u)} \geq-y, \tau(u)<\infty\right] \\
= & \frac{h_{i}}{h_{j}} e^{-\gamma u} \mathbb{E}_{L, i}\left[e^{\gamma R_{\tau(u)}} ; J_{\tau(u)}=j, R_{\tau(u)-} \leq x, R_{\tau(u)} \geq-y\right] .
\end{aligned}
$$

Condition on $\tau(u)=t, J_{\tau(u)}=k$ and $R_{\tau(u)^{-}}=v$, a claim that triggers ruin has distribution

$$
\frac{B_{L, k}(y)-B_{L, k}(v)}{1-B_{L, k}(v)}
$$

for $y \geq v$ under $\mathbb{P}_{L, i}$ and hence

$$
\begin{aligned}
& \mathbb{E}_{L, i}\left[e^{\gamma R_{\tau(u)}} ; J_{\tau(u)}=j, R_{\tau(u)-} \leq x, R_{\tau(u)} \geq-y \mid \tau(u)=t,\right. \\
& \left.J_{\tau(u)}=k, R_{\tau(u)-}=v\right] \\
= & 1(j=k, v \leq x) \int_{v}^{v+y} e^{-\gamma(z-v)} \frac{B_{L, k}(\mathrm{~d} z)}{1-B_{L, k}(v)} \\
= & 1(j=k, v \leq x) e^{\gamma v} \frac{\int_{v}^{v+y} e^{-\gamma z} e^{\gamma z} B_{k}(\mathrm{~d} z)}{\left[1-B_{L, k}(v)\right] \hat{B}_{k}(\gamma)} \\
= & 1(j=k, v \leq x) e^{\gamma v} \frac{B_{k}(v+y)-B_{k}(v)}{\int_{v}^{\infty} e^{\gamma z} \frac{B_{k}(\mathrm{~d} z)}{\hat{B}_{k}(\gamma)} \cdot \hat{B}_{k}(\gamma)} \\
= & 1(j=k, v \leq x) \frac{B_{k}(v+y)-B_{k}(v)}{\int_{v}^{\infty} e^{\gamma(z-v)} B_{k}(\mathrm{~d} z)} .
\end{aligned}
$$

Let $H_{i k}(x, t)=\mathbb{P}_{L, i}\left(J_{\tau(u)}=k, R_{\tau(u)-} \leq x, \tau(u) \leq t\right)$. Then

$$
\begin{aligned}
& F_{i j}(u, x, y) \\
= & \frac{h_{i}}{h_{j}} e^{-\gamma u} \sum_{k \in \mathcal{M}} \int_{0}^{\infty} \int_{0}^{\infty} 1(j=k, v \leq x) \frac{B_{k}(v+y)-B_{k}(v)}{\int_{v}^{\infty} e^{\gamma(z-v)} B_{k}(\mathrm{~d} z)} H_{i k}(\mathrm{~d} v, \mathrm{~d} t) \\
= & \frac{h_{i}}{h_{j}} e^{-\gamma u} \sup _{0 \leq v \leq x}\left[\frac{B_{j}(v+y)-B_{j}(v)}{\int_{v}^{\infty} e^{\gamma(z-v)} B_{j}(\mathrm{~d} z)}\right] \cdot \int_{0}^{\infty} \int_{0}^{\infty} H_{i j}(\mathrm{~d} v, \mathrm{~d} t)
\end{aligned}
$$




$$
\begin{aligned}
& =\frac{h_{i}}{h_{j}} e^{-\gamma u} \sup _{0 \leq v \leq x}\left[\frac{B_{j}(v+y)-B_{j}(v)}{\int_{v}^{\infty} e^{\gamma(z-v)} B_{j}(\mathrm{~d} z)}\right] \cdot \mathbb{P}_{L, i}\left(J_{\tau(u)}=j, R_{\tau(u)-} \leq x\right) \\
& =\frac{h_{i}}{h_{j}} e^{-\gamma u} \sup _{0 \leq v \leq x} \frac{B_{j}(v+y)-B_{j}(v)}{\int_{v}^{\infty} e^{\gamma(z-v)} B_{j}(\mathrm{~d} z)}
\end{aligned}
$$

which is the Lundberg-type upper bound for $F_{i j}(u, x, y)$.

Similarly,

$$
\begin{aligned}
& \mathbb{E}_{L, i}\left[\frac{e^{\gamma R_{\tau(u)}}}{h_{J_{\tau(u)}}} ; R_{\tau(u)-} \leq x, R_{\tau(u)} \geq-y \mid \tau(u)=t, J_{\tau(u)}=k, R_{\tau(u)-}=v\right] \\
= & \frac{1(v \leq x)}{h_{k}} \frac{B_{k}(v+y)-B_{k}(v)}{\int_{v}^{\infty} e^{\gamma(z-v)} B_{k}(\mathrm{~d} z)}
\end{aligned}
$$

and by the tower property of expectation,

$$
\mathbb{E}_{L, i}\left[\frac{e^{\gamma R_{\tau(u)}}}{h_{J_{\tau(u)}}} ; R_{\tau(u)-} \leq x, R_{\tau(u)} \geq-y\right] \leq \max _{j \in \mathcal{M}} \frac{1}{h_{j}} \sup _{0 \leq v \leq x} \frac{B_{j}(v+y)-B_{j}(v)}{\int_{v}^{\infty} e^{\gamma(z-v)} B_{j}(\mathrm{~d} z)}
$$

Hence

$$
F_{i}(u, x, y) \leq h_{i} e^{-\gamma u} \max _{j \in \mathcal{M}} \frac{1}{h_{j}} \sup _{0 \leq v \leq x} \frac{B_{j}(v+y)-B_{j}(v)}{\int_{v}^{\infty} e^{\gamma(z-v)} B_{j}(\mathrm{~d} z)}
$$

Theorem 2. Assume the net profit condition (2) holds and the adjustment coefficient $\gamma$ exists. The two-sided Lundberg bound for the distribution of the deficit at ruin starting with initial surplus $u$ and state of economy $i$ is given by

$$
\begin{gathered}
h_{i} e^{-\gamma u} \min _{j \in \mathscr{M}} \frac{1}{h_{j}} \inf _{v \geq 0} \frac{B_{j}(v+y)-B_{j}(v)}{\int_{v}^{\infty} e^{\gamma(z-v)} B_{j}(\mathrm{~d} z)} \leq F_{i}(u, \infty, y) \\
\leq h_{i} e^{-\gamma u} \max _{j \in \mathscr{M}} \frac{1}{h_{j}} \sup _{v \geq 0} \frac{B_{j}(v+y)-B_{j}(v)}{\int_{v}^{\infty} e^{\gamma(z-v)} B_{j}(\mathrm{~d} z)}
\end{gathered}
$$

and the two-sided Lundberg bound for the deficit at ruin starting with initial surplus $u$ and state of economy $i$ is given by 


$$
\begin{gathered}
h_{i} e^{-\gamma u} \min _{j \in \mathcal{M}} \frac{1}{h_{j}} \inf _{v \geq 0} \frac{1-B_{j}(v+y)}{\int_{v}^{\infty} e^{\gamma(z-v)} B_{j}(\mathrm{~d} z)} \leq \mathbb{P}_{i}\left(\tau(u)<\infty,-R_{\tau(u)}>y\right) \\
\leq h_{i} e^{-\gamma u} \max _{j \in \mathcal{M}} \frac{1}{h_{j}} \sup _{v \geq 0} \frac{1-B_{j}(v+y)}{\int_{v}^{\infty} e^{\gamma(z-v)} B_{j}(\mathrm{~d} z)} .
\end{gathered}
$$

Proof: From the proof of Theorem 1,

$$
\begin{aligned}
& \mathbb{E}_{L, i}\left[\frac{e^{-\gamma R_{\tau(u)}}}{h_{J_{\tau}(u)}} ; R_{\tau(u)} \geq-y \mid \tau(u)=t, J_{\tau(u)}=k, R_{\tau(u)-}=v\right] \\
= & \frac{1}{h_{k}} \frac{B_{k}(v+y)-B_{k}(v)}{\int_{v}^{\infty} e^{\gamma(z-v)} B_{k}(\mathrm{~d} z)},
\end{aligned}
$$

which shows the first result.

The second result follows by a similar argument.

Letting $y$ goes to infinity in the first result of Theorem 2, the two-sided Lundberg bound for the ruin probability same as that of Theorem VI.3.11 in Asmussen (2000) is obtained.

\section{NUMERICAL ILLUSTRATION}

For a simple illustration, we calculate the two-sided bounds for the distribution of the deficit at ruin and compare them with the theoretical value.

Consider a Markov-modulated risk model with three states of economy. Suppose that

$$
\boldsymbol{\Lambda}=\left[\begin{array}{ccc}
-\frac{1}{3} & \frac{1}{9} & \frac{2}{9} \\
\frac{1}{9} & -\frac{1}{3} & \frac{2}{9} \\
\frac{1}{6} & 0 & -\frac{1}{6}
\end{array}\right] \text { and } \beta=\left[\begin{array}{c}
\frac{1}{2} \\
\frac{1}{3} \\
1
\end{array}\right]
$$

In state of economy 1 , the claims sizes are exponentially distributed with mean 1 . In state of economy 2, the claim sizes are exponentially distributed with mean 6 . In state of economy 3 , the claim sizes are hyperexponentially distributed with two channels and the density is

$$
\frac{3}{4} e^{-x}+\frac{1}{2} e^{-2 x} .
$$

The stationary distribution of the continuous-time Markov chain is found to be

$$
\pi=\left[\begin{array}{lll}
\frac{9}{28} & \frac{3}{28} & \frac{4}{7}
\end{array}\right]
$$


and $\sum_{i=1}^{3} \pi_{i} \beta_{i} \mu_{B_{i}}=\frac{7}{8}$ which means that the relative security loading is $\frac{1}{7}$. The unique positive solution of the equation $\kappa(\alpha)=0$ is $\gamma=0.038215$. The right eigenvector $\boldsymbol{h}=\boldsymbol{h}^{(\gamma)}$ with the normalization $\boldsymbol{\pi} \boldsymbol{h}^{(\gamma)}=1$ is

$$
\boldsymbol{h}=\left[\begin{array}{l}
0.989849 \\
1.194539 \\
0.969234
\end{array}\right]
$$

For phase-type claims closed-form solution of $F_{i}(u, \infty, y)$ has been obtained by $\mathrm{Ng}$ and Yang (2005).

The following shows the theoretical value and the two-sided Lundberg bounds for some combinations of $u, y$ and $i$.

For $u=1, i=1$ :

\begin{tabular}{cccc}
\hline \hline$y$ & $F_{1}(1, \infty, y)$ & lower bound & upper bound \\
\hline 0.5 & 0.20586 & 0.04731 & 0.41435 \\
1 & 0.33604 & 0.09083 & 0.63118 \\
2 & 0.47964 & 0.16771 & 0.81742 \\
4 & 0.59543 & 0.28789 & 0.90178 \\
6 & 0.64902 & 0.37310 & 0.91272 \\
8 & 0.68346 & 0.43569 & 0.91419 \\
\hline \hline
\end{tabular}

For $u=2, i=3$ :

\begin{tabular}{cccc}
\hline \hline$y$ & $F_{3}(2, \infty, y)$ & lower bound & upper bound \\
\hline 0.5 & 0.20936 & 0.04632 & 0.40572 \\
1 & 0.33900 & 0.08894 & 0.61804 \\
2 & 0.47854 & 0.16421 & 0.80039 \\
4 & 0.58635 & 0.28189 & 0.88300 \\
6 & 0.63412 & 0.36621 & 0.89371 \\
8 & 0.66441 & 0.42662 & 0.89515 \\
\hline \hline
\end{tabular}

For $u=6, i=1$ :

\begin{tabular}{cccc}
\hline \hline$y$ & $F_{1}(6, \infty, y)$ & lower bound & upper bound \\
\hline 0.5 & 0.09430 & 0.04060 & 0.35562 \\
1 & 0.16044 & 0.07795 & 0.54171 \\
2 & 0.24777 & 0.14394 & 0.70155 \\
4 & 0.34771 & 0.24708 & 0.77395 \\
6 & 0.40952 & 0.32098 & 0.78334 \\
8 & 0.45249 & 0.37393 & 0.78460 \\
\hline \hline
\end{tabular}


For $u=10, i=2$ :

\begin{tabular}{cccc}
\hline \hline$y$ & $F_{2}(10, \infty, y)$ & lower bound & upper bound \\
\hline 0.5 & 0.07629 & 0.04205 & 0.36832 \\
1 & 0.13330 & 0.08074 & 0.56107 \\
2 & 0.21520 & 0.14908 & 0.72661 \\
4 & 0.31998 & 0.25591 & 0.80160 \\
6 & 0.38882 & 0.33245 & 0.81136 \\
8 & 0.43731 & 0.38729 & 0.81263 \\
\hline \hline
\end{tabular}

As in the case of the two-sided Lundberg bounds for the ruin probability, the bounds are not very tight because of the various supremums and infimums. But it can be seen that the bounds still give a rough idea of the true value in many cases.

\section{ACKNOWLEDGMENTS}

The work described in this paper was partially supported by a grant from the Research Grants Council of the Hong Kong Special Administrative Region, China (Project No. HKU 7239/04H). The authors would like to thank the referee for careful reading of the paper and helpful suggestions that improved the presentation of the paper.

\section{REFERENCES}

Asmussen, S. (1989) "Risk theory in a Markovian environment". Scandinavian Actuarial Journal, 1989, 69-100.

Asmussen, S. (2000) Ruin Probabilities. World Scientific.

Asmussen, S. (2003) Applied Probability and Queues. 2nd ed., New York: Springer-Verlag.

Dickson, D.C.M. (1992) "On the distribution of the surplus prior to ruin", Insurance: Mathematics and Economics, 11: 191-207.

Dickson, D.C.M. and Dos Reis, A.E. (1994) "Ruin problems and dual events", Insurance: Mathematics and Economics, 14: 51-60.

Dufresne, F. and Gerber, H.U. (1988) "The surpluses immediately before and at ruin, and the amount of the claim causing ruin", Insurance: Mathematics and Economics, 7: 193-199.

Gerber, H.U. (1973) "Martingales in risk theory", Mitteilunger der Schwerizerischen Vereinigung der Versicherungsmathematiker, 205-216.

Gerber, H.U. (1979) An Introduction to Mathematical Risk Theory. S.S. Huebner Foundation Monograph Series No. 8, R. Irwin, Homewood, IL.

Gerber, H.U., Goovaerts, M.J. and KaAs, R. (1987) "On the probability and severity of ruin", ASTIN Bulletin, 17: 151-163.

Gerber, H.U. and Shiu, E.S.W. (1998) "On the time value of ruin", North American Actuarial Journal, 2(1): 48-72.

Gerber, H.U. and ShiU, E.S.W. (1997) "The joint distribution of the time of ruin, the surplus immediately before ruin, and the deficit at ruin", Insurance; Mathematics and Economics, 21: $129-137$.

Gerber, H.U. and ShiU, E.S.W. (2005) "The time value of ruin in a Sparre Andersen Model", North American Actuarial Journal, 9(2): 49-84. 
Grandell, J. (1990) Aspects of Risk Theory. Springer-Verlag.

NG, A.C.Y. and YANG, H. (2005) "On the joint distribution of surplus prior and after ruin for a Markovian regime-switching model", Submitted.

Rolski, T., Schmidli, H., Schmidt, V. and Teugels, J. (1999) Stochastic Processes for Insurance and Finance. Wiley.

SCHMIDLI, H. (1999) "On the distribution of the surplus prior and at ruin", ASTIN Bulletin, 29: 227-244.

Andrew C.Y. NG And Hailiang YANG

Department of Statistics and Actuarial Science

The University of Hong Kong

Pokfulam Road, Hong Kong 